\title{
Using Telemedicine for Providing Supportive and Palliative Care Patients with Advanced Cancer During The COVID-19 Pandemic in Ukraine
}

\author{
Hojouj Mohammad IM*, N Bondarenko, Zavizion VF and Procach A Hojouj T Elhaj M \\ SE "Dnipropetrovsk medical academy of Health Ministry of Ukraine" Department of oncology and medical radiology Volodymyr Vernadskii \\ str, 9, Dnipro, 49044, Ukraine.
}

*Corresponding author: Sánchez Enríquez S MD, PhD and Briseño Ramírez J MD, specialist in internal medicine and infectology, Department of Clinics, Los Altos University Center, University of Guadalajara, Jalisco, Mexico.

\begin{abstract}
COVID-19 has overwhelmed the capacity of health care systems, limiting access to supportive and palliative care for patients with advanced cancer. Telemedicine has emerged as a tool to provide care continuity to patients while limiting the risk of contagion. However, implementing telemedicine in resource-limited settings is challenging. We report the results of a multidisciplinary patient-navigator-led telemedicine supportive care program in Dnipro City. One-hundred sixty-five telemedicine interventions were provided to 50 patients (median age 67, 47\% female). A quarter of the patients had less than or equal to elementary school education, and $18 \%$ lived in a rural area. The most com-mon interventions were psychological care (30\%), pain and symptom control (27\%), and nutritional counseling (10\%). Half of the interventions were pro-vided by video conferencing. The most common patient-reported barrier was limited experience using communication technology. Our results demonstrate the feasibility of providing supportive and palliative care interventions using telemedicine in resource-limited settings.
\end{abstract}

\section{Introduction}

On March 11, the World Health Organization declared COVID-19 a pandemic [1]. As of June 23, >9 million cases and 475,000 deaths had been reported worldwide [2]. In Ukraine , COVID-19 led to a health care system reorganization [3]: some hospitals were designated as COVID-19 centers, and as a consequence, outpatient clinics were closed, elective surgeries were suspended, and patients needing emergency care were referred to non-COVID hospitals. State Establishment "Dnipropetrovsk Medical Academy of Health Ministry of Ukrainis a public academic hospital No 4 In Dnipro City, which was converted to a COVID-19 center on March 25, 2020. Patients undergoing active cancer treatment at maintained their oncology clinic visits and treatment appointments, although all other supportive care visits were suspended. Offering continued supportive and palliative care represented a challenge, and telemedicine emerged as a potential solution [3]. Telemedicine is the use of communication and information technologies to provide clinical services [4] and represents an option to provide remote support to patients during the pandemic [5]. However, implementing telemedicine in resource-limited settings might be challenging owing to disparities in access [6]. Here, we describe how we transformed a multidisciplinary supportive and palliative care program in-to a telemedicine intervention for patients with advanced cancer during the COVID-19 pandemic.

\section{Materials and Methods}

In 2019, we implemented “Te Acompañamos," a patient navigation program to increase access to supportive and palliative care for patients with advanced cancer at Oncology department DMA . Currently, all consecutive patients with a recent diagnosis of advanced cancer seen at the oncology clinics at Oncology department DMA are included in Te Acompañamos and undergo basal supportive and palliative care needs assessments (depression, anxiety, fatigue, pain, malnutrition, caregiver burden), which are reviewed by a multidisciplinary patient navigator (PN)-led team 
(palliative care, physical therapy, geriatrics, psychology/psychiatry, oncology, and nutrition) that recommends tailored interventions. The PN then helps patients obtain the required interventions and engage in advance care planning, including advance directive completion. Up until Oncology department DMA conversion to a COVID-19 center, Te Acompañamos had provided care for 431 patients with advanced cancer.

Upon Oncology department DMA conversion, we undertook adaptations to allow for Te Acompañamos to continue using telemedicine:

a) supportive care needs assessments were administered remotely.

b) the multidisciplinary team met weekly using Zoom to discuss patient needs and proposed interventions.

c) the PN presented patients with intervention plans remotely and

d) if the interventions were accepted, team members implemented them remotely using various contact methods (video conferences, phone calls, messaging, etc.). All the patients with a recent diagnosis of advanced solid cancer who were already enrolled in Te Acompañamos were candidates for telemedicine interventions. In addition, new patients seen at the oncology clinics after Oncology department DMA conversion and who had a diagnosis of advanced cancer in the previous 3 months were also included. Before the interventions were implemented, patients were contacted to assess the availability of communication methods and received a brief training on how to use them. If needed, e-mail accounts were created for them to receive recommendations and materials. Patients' privacy and security were protected by instructing providers regarding the best clinical practice for telemedicine. For example, telemedicine interventions were recommended to occur in private settings, headphone use was encouraged, patients were advised to avoid high-tone speakers, and recording was not allowed (both for the provider and for the patient). In addition, only non-public-facing communication products using end-to-end encryption were used (Zoom, Google Hangouts video, or WhatsApp video chat).

After each intervention, providers were prospectively asked to complete an online multiple-choice qualitative survey designed to assess barriers for telemedicine implementation. The survey was composed of 12 items (supplemental online Appendix 1), which included open-ended questions for describing the intervention (type, cause of intervention, method used, length, and supportive material used), the findings during the consultation, and any follow-up plans. Providers also answered whether the patient needed support to complete the intervention, who provided that support, and what barriers were identified before and during the intervention. Approval from the institutional review board at INCMNSZ was obtained. We recorded the number and type of interventions that were pro-vided remotely, as well as the communication methods used. In addition, we assessed potential barriers for successful implementation of the interventions. Descriptive statistics including means, medians, and standard deviations were used to analyze the data.

\section{Results}

Between March 01 and June 30, 153 supportive care interventions were provided to 40 patients (median number of interventions per patient 3; range 1-12). Median age was 66 years (range 33-90), and 55.7\% were female[7]. More than half (52.4\%) had less than or equal to high school education $(26.6 \%$ less than or equal to elementary school). The most common tumors were gastrointestinal ( $n=21,48 \%$ ), followed by breast ( $n=5,10 \%$ ). Supportive care needs more commonly prompting interventions were pain $(\mathrm{n}=51 ; 31.7 \%)$; anxiety $(\mathrm{n}=30 ; 18 \%)$, fatigue $(\mathrm{n}=28$; $16.7 \%)$, depression ( $\mathrm{n}=23 ; 13.7 \%)$, and malnutrition $(\mathrm{n}=19$; $11.3 \%)$. Others included nausea $(\mathrm{n}=12 ; 7.3 \%)$, caregiver burden ( $n=11 ; 6.7 \%)$, abnormal geriatric screening ( $n=8 ; 4.9 \%)$, and potential suicidal ideation $(n=4 ; 2.4 \%)$.

\section{Discussion}

During the COVID-19 pandemic, and despite the low availability of resources, the Te Acompañamos multidisciplinary supportive and palliative care program has provided a significant number of telemedicine interventions, even for patients with significant symptom burden ( $\geq 3$ supportive care needs). Telemedicine represents an effective modality for delivering supportive and palliative care [8]. However, implementing a telemedicine program in resource-limited settings might be challenging owing to policy issues, concerns regarding malpractice-related issues, lack of formal organizational structures and reimbursement mechanisms, poor interest from health care providers, technological barriers, prescription issues, and social/cultural limitations. However, we show that telemedicine interventions could be provided even though a significant proportion of our patients had low educational levels, lived in rural area, and/or were older adults [5]. The most common barriers reported by the providers were technological, including poor Internet connection and low technology awareness. Privacy issues also were reported, particularly during psychological care interventions, because patients often needed assistance from caregivers to maintain communication. In Mexico, the average number of people per household is four [9], and this overcrowding may contribute to a lack of privacy during real-time telemedicine interventions. The increasing use of telemedicine during the COVID-19 pandemic has raised concerns be-cause some ways of remote communication between patients and providers might not fully comply with privacy regulations. In that regard, a 
recent notification by the U.S. Department of Health and Human Services recommends ensuring privacy by using non-public-facing platforms and considers it acceptable to use software with end-to end encryption that allows only individual users to communicate through it, such as Microsoft Teams, Updox, VSee, Zoom, Google Suite Hangouts, Cisco Webex Meeting, WhatsApp video chat, etc. [10]. That being said, it is important to mention that only providerreported barriers were collected in this study and that patientreported barriers could potentially differ, particularly regarding the patients' opinion on privacy issues. For telemedicine to work, patients must have access to electronic devices and to the Internet, which might be challenging in low-income populations. However, although a significant proportion of people in Mexico have limited incomes, and $<50 \%$ of Mexicans own a computer, a significant proportion (75\%) have an Internet connection and $>90 \%$ own a smartphone. In our study, the vast majority of patients received telemedicine interventions through a smartphone video conference or phone call [11].

\section{Conclusion}

Telemedicine program for patients with advanced cancer during the COVID-19 pandemic is feasible in low- and middleincome countries using readily available resources Implementing a supportive and palliative care. Telemedicine represents an excellent method to maintain care continuity for patients with high symptom burden while limiting the risk of contagion for both patients and providers.

\section{References}

1. World Health Organization. WHO Timeline -COVID-19.

2. (2020) Johns Hopkins University. COVID-19 Dashboard by the Center for Systems Science and Engineering (CSEE) at Johns Hopkins University (JHU).

3. (2020) Confirman el pricer caso de coronavirus en Mexico. BBC News Mundo.

4. (2020) Cancer Patient Management during the COVID-19 Pandemic. ESMO guidelines.

5. Bali S (2020) Barriers to Development of Telemedicine in Developing Countries.

6. (2020) ASCO Special report: A guide to cancer care delivery during the COVID-19 Pandemic.

7. Eberly LA, Khatana SAM, Nathan AS (2020) Telemedicine outpatient cardiovascular care during the COVID-19 pandemic: Bridging or opening the digital divide? Circulation 142: 510- 512.

8. Calton B, Abedini N, Fratkin M (2020) Telemedicine in the Time of Coronavirus. J Pain Symptom Manage 60: e12- e14.

9. (2020) Instituto Nacional de Estadística y Geografía (INEGI). Vivienda.

10. (2020) Notification of enforcement discretion for telehealth remote communications during the COVID-19 nationwide public health emergency. US Department of Health and Human Services.
(C) (P) This work is licensed under Creative

To Submit Your Article Click Here:

Submit Article
DOI: $10.32474 /$ RRHOAJ.2021.06.000230

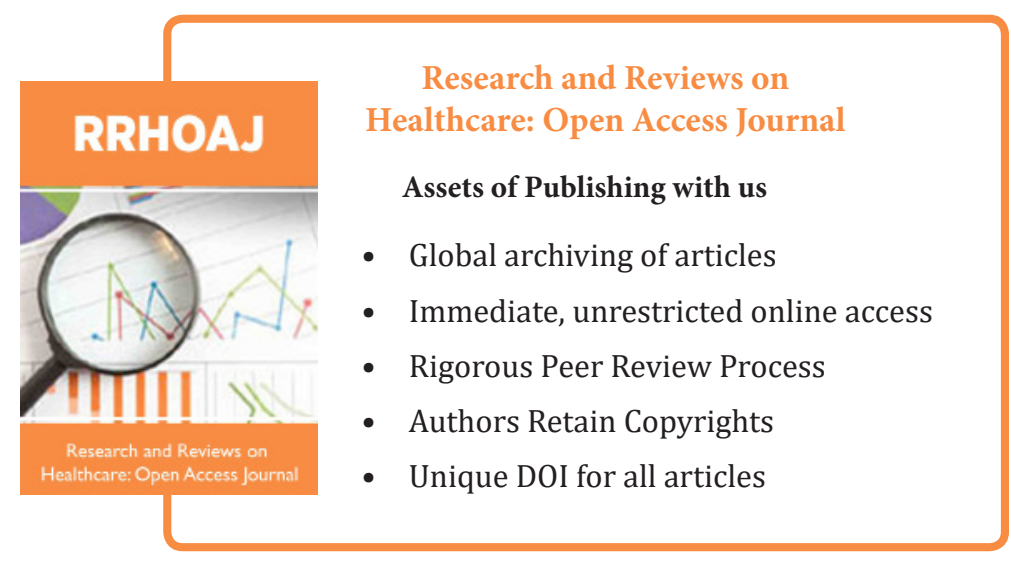

